\title{
Pacific
}

Journal of

Mathematics

\section{THE UNIQUENESS OF COMPACT CORES FOR 3-MANIFOLDS}

\section{LuKe HARRis AND Peter ScotT}




\title{
THE UNIQUENESS OF COMPACT CORES FOR 3-MANIFOLDS
}

\author{
Luke Harris and Peter ScotT
}

\begin{abstract}
A compact core for a 3-manifold $M$ is a compact sub-manifold $N$ of $M$ whose inclusion in $M$ induces an isomorphism of fundamental groups. A uniqueness result for compact cores of orientable 3-manifolds is known. The authors show that compact cores are not unique in any reasonable sense for non-orientable 3-manifolds, but they prove a finiteness result about the number of possible cores.
\end{abstract}

If $M$ is a non-compact 3-manifold with finitely generated fundamental group, then Scott showed in [Sc1] that there is a compact sub-manifold $N$ of $M$ with the natural map $\pi_{1}(N) \rightarrow \pi_{1}(M)$ an isomorphism. See [R-S] for a simpler proof. We call such a sub-manifold a core or compact core for $M$. In [McC-Mi-Sw], McCullough, Miller and Swarup showed that if $N_{1}$ and $N_{2}$ are irreducible compact cores of a $\mathbb{P}^{2}$-irreducible 3-manifold $M$, then $N_{1}$ and $N_{2}$ are homeomorphic. In this paper, we seek to generalize this to the case when $M$ and its compact cores have no irreducibility restrictions. Of course, we cannot any longer expect to prove that two cores of $M$ are homeomorphic, because the Poincare conjecture is not resolved. Thus one core for $M$ might be the connected sum of another core with a homotopy sphere. Also we can obtain new cores by removing a 3-ball from a core or by replacing a connected summand of a core which is a 2-sphere bundle over the circle by a disc bundle over the circle. However, we give an example showing that even if one works modulo the equivalence relation on cores generated by the above operations then uniqueness does not hold. We also show that there are only finitely many different cores in a given 3-manifold up to the equivalence relation of almost homeomorphism which we define in $\S 1$. We end by using this finiteness result to prove a natural finiteness result for the boundary of a 3-manifold which has finitely generated fundamental group. The result is the following.

Theorem 3.2. Let $M$ be a 3-manifold with finitely generated fundamental group. Then

(i) There are only finitely many boundary components $F$ of $M$ with $\operatorname{Im}\left(\pi_{1}(F) \rightarrow \pi_{1}(M)\right)$ not trivial or infinite cyclic, 
(ii) There are only finitely many boundary components $F$ of $M$ with $\operatorname{Im}\left(\pi_{1}(F) \rightarrow \pi_{1}(M)\right)$ infinite cyclic and with essential core a Möbius band,

(iii) Of those components of the boundary $F_{i}$ with $\operatorname{Im}\left(\pi_{1}\left(F_{i}\right) \rightarrow \pi_{1}(M)\right)$ infinite cyclic and with essential core an annulus, there are only finitely many conjugacy classes in $\pi_{1}(M)$ of $\operatorname{Im}\left(\pi_{1}\left(F_{i}\right) \rightarrow \pi_{1}(M)\right)$.

In a separate paper [H-S], we use Theorem 3.2 to extend earlier results of Brin, Johannson and Scott [BJS] on compact totally peripheral 3-manifolds to the non-compact case.

The work in this paper is part of the Liverpool Ph.D. thesis of Luke Harris completed under the supervision of Peter Scott in 1988. Since then Harris obtained a job not in the academic world and has never had time to prepare this for publication. Finally, Scott agreed to prepare this for publication, to avoid the complete disappearance of his work.

\section{$\S 1$. Preliminaries and the example.}

Definition. Let $M$ and $N$ be compact 3-manifolds. Then $M$ and $N$ are almost homeomorphic if they are homeomorphic up to connected sum with compact simply connected manifolds (3-balls and fake 3-spheres) and up to replacing $\mathbb{P}^{2} \times I$ 's with fake $\mathbb{P}^{2} \times I$ 's.

We start with our example to show non-uniqueness for cores. Let $M$ be a 3-manifold with finitely generated fundamental group, and with core $N$ in $\stackrel{\circ}{M}$. Suppose that $M-\stackrel{\circ}{N}$ has at least two components $R_{1}$ and $R_{2}$, and let $F_{1}$ and $F_{2}$ be the components of $\partial N$ which lie in $R_{1}$ and $R_{2}$. Let $X$ denote the solid torus. Note that similar examples can be constructed if $X$ is any compact manifold with at least one boundary component not the 2-sphere. We can form the connected sum $M \# X$ in several ways, depending on the choice of 3-balls in $M$ and in $X$. However, $M \# X$ is independent of this choice.

If we form $M \# X$ by selecting a 3-ball in $M$ which lies in the region $R_{1}$, then a natural selection of core $N_{1}$ for $M \# X$ is $N$ with a 1-handle attached to $F_{1}$. If we select the 3 -ball in $R_{2}$, then the core $N_{2}$ could be $N$ with a 1 -handle attached to $F_{2}$. We could also select the 3 -ball to lie in $N$, in which case the natural core $N_{0}$ would be $N \# X$. Note that this is homeomorphic to $N$ with the interior of a trivial solid torus removed, where trivial means that the solid torus lies in a 3-ball in $N$ and is unknotted there. Also note that $N_{1}$ and $N_{2}$ are each a boundary connected sum of $N$ and $X$.

So long as $F_{1}$ and $F_{2}$ are not homeomorphic, and not 2-spheres, these three cores are non-homeomorphic in a fairly non-trivial way. They have different 
boundary, for example, and the difference in boundary is not caused just by adding or removing 2-sphere components. Thus they are not almost homeomorphic. Further, so long as $F_{1}$ and $F_{2}$ have genus at least two, these cores cannot be equivalent under the coarser equivalence relation obtained from the operation of replacing a summand which is a sphere bundle over the circle by a solid torus. However, they do have certain similarities. All contain pieces homeomorphic with $X$ and $N$, at least up to connected sum with 3-balls, and these pieces are connected by 1 -handles and $S^{2} \times I$ 's.

We cannot avoid this problem even if we insist that one of the cores be embedded in the other, since in the example given it is possible to embed either of $N_{1}$ and $N_{2}$ inside $N_{0}$. To see how to embed $N_{1}$ in $N_{0}$, for example, consider a simple closed curve $\alpha$ on $F_{1}$ which bounds a disc $D$ in $F_{1}$ containing the endpoints of the 1-handle of $N_{1}$. We can also find a disc $E$ in $R_{1}$ with boundary $\alpha$, such that $E \cup D$ defines a 2-sphere in $R_{1}$ which is parallel to the boundary of the 3 -ball we removed from $R_{1}$ to form $M \# X$. Then $N_{1}$ together with a regular neighbourhood of $E$ is homeomorphic to $N$ minus a trivial solid torus and hence homeomorphic to $N_{0}$.

It is clear that this example is not a special case, and that any connected sum between two 3-manifolds with cores having non-spherical boundary may have a number of non-homeomorphic cores, constructed similarly to $N_{0}, N_{1}$ and $N_{2}$ above.

Now we will need a few definitions. The first four are after Scott in [Sc2]. Definition. A sub-manifold $X$ of a 3-manifold $M$ is incompressible if $\partial X$ is incompressible in $M$.

Remark. Then the natural map $\pi_{1}(X) \rightarrow \pi_{1}(M)$ is injective.

Definition. A 3-manifold $N$ is weakly irreducible if the manifold $\hat{N}$ obtained from $N$ by attaching a 3-ball to every boundary 2-sphere of $N$ is irreducible.

Definition. A chunk in a 3-manifold is a sub-manifold $X$ of $M$ which is connected, compact, incompressible and weakly irreducible.

Remark. With this definition, a (punctured) 2-sphere bundle over the circle is not a chunk.

Definition. Let $M$ be a 3-manifold, with fundamental group $G=\pi_{1}(M)$, and let $H$ be a finitely generated indecomposable subgroup of $G$. Then a chunk in $M$ for $H$ is a chunk $X$ in $M$ such that $\pi_{1}(X)$ contains a conjugate (in $G$ ) of $H$.

Observe that if $N$ is a compact core of a 3-manifold $M$, then we can decompose $N$ into chunks by cutting along a maximal family of 2 -spheres embedded in $N$ and then cutting along compressing discs for the boundaries of the resulting pieces. Thus $N$ can be viewed as a collection of chunks 
embedded in $M$ joined together by 1-handles and $S^{2} \times I$ 's.

Clearly if $\pi_{1}(M)=F_{r} * G_{1} * G_{2} * \ldots * G_{s}$ where $F_{r}$ is free and the $G_{i}$ are indecomposable and not infinite cyclic, then there must be exactly $s$ chunks $X_{i}$ for a core $N$ that have non trivial fundamental group, with $\pi_{1}\left(X_{i}\right)=G_{i}$ up to conjugacy after reordering. Thus the non trivial chunks for any two cores are in 1-1 correspondence. The main result of this section is that these chunks are almost homeomorphic.

Theorem 1.1. Let $X^{\prime}$ and $X$ be chunks in a 3-manifold $M$ with finitely generated fundamental group, and suppose that $\pi_{1}\left(X^{\prime}\right)$ and $\pi_{1}(X)$ are both conjugate to $H$, an indecomposable factor not $\mathbb{Z}$ in a free product decomposition of $\pi_{1}(M)$. Then $X^{\prime}$ and $X$ are almost homeomorphic.

Remark. Such chunks cannot contain fake 3-balls, but may contain fake $\mathbb{P}^{2} \times I$ 's.

As a first step, we prove the following special case of Theorem 1.1.

Lemma 1.2. The result of Theorem 1.1 holds if either $X$ and $X^{\prime}$ are disjoint or if one lies in the interior of the other.

Proof. First consider the case when $X$ lies in the interior of $X^{\prime}$. We know that $\pi_{1}(X)$ and $\pi_{1}\left(X^{\prime}\right)$ are both conjugate to $H$, and that $\pi_{1}(X)$ is a subgroup of $\pi_{1}\left(X^{\prime}\right)$. But $H=\pi_{1}(X)$ is an indecomposable factor of $\pi_{1}(M)$, and so no conjugate of $H$ can be properly contained in $H$. Thus we deduce that the inclusion of $X$ in $X^{\prime}$ induces an isomorphism of fundamental groups.

Consider a component $R$ of $X^{\prime}-\stackrel{\circ}{X}$. Clearly $R \cap X=F$, a single boundary component of $X$. But we must also have $\pi_{1}(R)=\pi_{1}(F)$ by van Kampen's theorem, since $\pi_{1}\left(X^{\prime}\right)=\pi_{1}(X)$. Then the $h$-cobordism theorem tells us that $R$ must be homeomorphic to $F \times I$ connected sum with 3 -balls unless $F \cong \mathbb{P}^{2}$, in which case $R$ might be a fake $\mathbb{P}^{2} \times I$ connected sum with 3 -balls. (Note that in fact we cannot have fake 3-balls in $R$, since non-simply connected chunks do not contain fake 3 -balls.) This is true for all components of $X^{\prime}-\stackrel{\circ}{X}$, and so we conclude that $X^{\prime}$ is almost homeomorphic to $X$.

Next consider the case when $X$ and $X^{\prime}$ are disjoint. Take the cover $M_{H}$ of $M$ with fundamental group $H$. Then $X$ and $X^{\prime}$ lift into $M_{H}$. Thus $H$ is the fundamental group of a graph of groups with $\pi_{1}(X)$ and $\pi_{1}\left(X^{\prime}\right)$ as vertex groups. As each of these vertex groups equals $H$, it follows that there is a path between these vertices with all edge and vertex groups equal to $H$. In particular, for $X$ and $X^{\prime}$ there are boundary components $F$ and $F^{\prime}$ respectively with $\pi_{1}(F) \rightarrow \pi_{1}(X)$ and $\pi_{1}\left(F^{\prime}\right) \rightarrow \pi_{1}\left(X^{\prime}\right)$ being isomorphisms. We apply the $h$-cobordism theorem to see that both $X$ and $X^{\prime}$ are almost 
homeomorphic to products $F \times I \cong F^{\prime} \times I$. Thus $X$ and $X^{\prime}$ must be almost homeomorphic. This completes the proof of Lemma 1.2.

Proof of Theorem 1.1. We may suppose that $\partial M$ is empty, by pushing $X$ and $X^{\prime}$ away from $\partial M$ and then deleting $\partial M$. Then we can find a compact sub-manifold $K$ with $X$ and $X^{\prime}$ embedded in the interior of $K$. The proof proceeds by altering $K$ in a fairly canonical way until we find a chunk $C_{1}$ derived from $K$ with $X$ embedded in $C_{1}$. Lemma 1.2 then shows that $X$ and $C_{1}$ must be almost homeomorphic. We may also alter $K$ in a slightly different way to obtain a chunk $C_{1}^{\prime}$ containing $X^{\prime}$, and so this chunk must be almost homeomorphic to $X^{\prime}$. But because we obtained the chunks in each case in a fairly standard way, we will be able to show that they must themselves be almost homeomorphic, which will complete the proof.

So for the moment, we will consider $X$ only. Consider a family of 2spheres $\Sigma$ embedded in $K$ corresponding to a prime decomposition of $K$. Note that the pieces obtained by splitting along any such family are unique up to homeomorphism and connected sum with 3-balls. In particular, the pieces are unique up to almost homeomorphism. We will alter $\Sigma$ so that it does not intersect $X$, but so that it remains a representation of a prime decomposition of $K$.

$\Sigma$ intersects $\partial X$ in a collection of embedded circles. $\partial X$ is incompressible, so we can choose an innermost circle $C$ of $\Sigma \cap \partial X$ bounding a disk $E$ in $\partial X$, with $E \cap \Sigma=\partial E=C$.

Now we cut and paste $\Sigma$ along $C$ using $E$, and push the new $\Sigma$ away from $\partial X$ on both sides. After deleting any redundant 2 -spheres, $\Sigma$ still represents a decomposition of $K$ into primes. We repeat until $\Sigma \cap \partial X$ is empty.

Some components of $\Sigma$ may lie inside $X$. We delete these components from $\Sigma$ and replace them with the spherical boundary components of $X$, and then again delete any redundant 2 -spheres. Since $X$ is weakly irreducible, any 2sphere embedded in $X$ other than a boundary sphere must be redundant, and thus $\Sigma$ still represents a decomposition of $K$ into primes. So we may cut $K$ along this new $\Sigma$, and $X$ will be contained in one of the pieces.

We now wish to compress the boundary of the pieces, whose union we will continue to call $K$. We do this by sequentially finding compressing discs for $\partial K$, lying in either $K$ or $M-\stackrel{\circ}{K}$. If a disc $D$ lies outside $K$, then we add a regular neighborhood of the disc to $K$. If the disc is contained in $K$, then we cut along it. Note that, so long as the boundaries of the discs and the order they are dealt with is the same, any sequence of compressing discs yields pieces unique up to homeomorphism and connected sum with 3-balls. This is because the components of $K$ are weakly irreducible, and any two discs with the same boundary embedded in an irreducible 3-manifold are isotopic. 
Consider a disc $D$ lying in $K$. Then $D$ intersects $\partial X$ in a collection of embedded circles. $\partial X$ is incompressible, so we can find an innermost circle $C$ bounding a disc $E$ in $\partial X$ with $D \cap E=\partial E=C$.

We cut and paste $D$ along $C$ using $E$, and discard the 2-sphere component of the result. Then we push $D$ away from $\partial X$,thus reducing the intersection number of $D$ with $\partial X$. We can repeat until $D \cap \partial X=\emptyset$ and thus $D \cap X=\emptyset$ since $\partial D \subset K-X$. Note that this does not disturb $\partial D$, so $D$ is still a compressing disc for $\partial K$ contained in $K$.

So after compressing the boundary of all the components, we have derived from the original $K$ a collection of chunks, one of which contains $X$. Call this chunk $C_{1}$. Lemma 1.2 shows that $X$ and $C_{1}$ are almost homeomorphic.

If there is another chunk $C_{2}$ derived from $K$ which also has fundamental group conjugate to $H$, then Lemma 1.2 again shows that it is also almost homeomorphic to $X$.

Now all the above construction can be done to find a number of chunks $C_{i}^{\prime}$ for $K$ corresponding to $X^{\prime}$. As we have already pointed out, we must get the same chunks up to homeomorphism and connected sum with 3-balls as we did with the first construction. Thus $C_{i}^{\prime}$ is almost homeomorphic to $C_{\jmath}$ for some $j$. It follows that $X$ is almost homeomorphic to $X^{\prime}$, which completes the proof of Theorem 1.1.

\section{$\S 2$. There are only finitely many compact cores for 3-manifold.}

In $\S 1$, we gave an example which rules out the possibility of a uniqueness result such as that to be found in the paper [McC-Mi-Sw] of McCullough, Miller and Swarup. In this section we show that, up to almost homeomorphism, there are only finitely many cores for any 3-manifold $M$.

Theorem 2.1. Let $M$ be a 3-manifold with finitely generated fundamental group. Then, up to almost homeomorphism, there are only finitely many different cores for $M$.

Proof. The proof uses the result of the previous section on uniqueness of non-simply connected chunks in a 3-manifold. Since all cores have a decomposition into essentially the same chunks, then these same chunks can be used as the building blocks to construct any core for $M$. We can then show that there are, up to almost homeomorphism, only finitely many ways to put these chunks together to give a compact 3-manifold with fundamental group equal to $\pi_{1}(M)$. Of course, if chunks were unique up to homeomorphism, it would be trivial that there could only be a finite number of cores up to homeomorphism. 
So first consider the decomposition of a compact core $N$ into chunks, by splitting along 2-spheres and discs. Then $N$ is the union of these chunks together with 1-handles and $S^{2} \times I$ 's. If $M$ has fundamental group $\pi_{1}(M)=$ $G=F_{r} * G_{1} * G_{2} * \cdots * G_{s}$ where $F_{r}$ is free of rank $r$ and the $G_{i}$ are indecomposable not infinite cyclic, then we can decompose $N$ into exactly $s$ chunks $C_{i}$ that are not simply connected, and such that $\pi_{1}\left(C_{i}\right)=G_{i}$ up to conjugacy in $G$. We apply the result of $\S 1$ to see that, up to almost homeomorphism, all cores for $M$ have exactly the same non simply connected chunks. It will be useful to choose our splitting family of spheres and discs to be minimal in the sense that no proper subfamily splits $N$ in this way.

Using the decomposition mentioned in the introduction, we first cut sequentially along 2-spheres $\left\{S_{j}\right\}$, and then along discs $\left\{D_{k}\right\}$. We can easily arrange that all the discs and spheres can be embedded in $N$, and are disjoint from one another. Thus the order in which we cut along the spheres and discs is irrelevant. Since the construction of a core from the chunks is essentially the reverse operation to that of cutting along the spheres and discs, we will find it useful to choose a particular order in which to decompose $N$.

We now wish to organize the decomposition into four steps. In step one, we cut along non-separating 2-spheres. In step two, we cut along discs which correspond to 1-handles attached to spherical boundary components of chunks. In step three, we cut along separating 2-spheres, and finally in step four we split along the remaining discs. We will comment on each stage of the decomposition.

Step 1 : Cutting along the non-separating 2-spheres.

Let $S$ be such a non-separating 2-sphere. Then we can find a regular neighborhood $S \times I$ for $S$. Since $S$ is non-separating, we cam find an arc $\lambda$ in the complement of $S \times I$ joining $S \times 0$ to $S \times 1$. $S \times I$ together with a regular neighborhood of $\lambda$ defines a punctured 2-sphere bundle over the circle. Thus every non-separating sphere in the family $S_{i}$ corresponds to a 2-sphere bundle over the circle in a prime decomposition of $N$.

Step 2: Cutting along discs corresponding to 1-handles attached to spherical boundary components of chunks.

This step is much simpler that it sounds. Let $D$ be a disc in the family $\left\{D_{k}\right\}$. We can cut along all the other spheres and discs in the decomposition leaving $D$ until last. Then $D$ corresponds to a 1-handle with ends attached to discs in the boundary of the chunks. Since $D$ is a compressing disc, if one end of the 1-handle is attached to a spherical boundary component $S$ of a chunk, it must be that the other end is also attached to $S$, since $S$ is separating and the family of discs $\left\{D_{k}\right\}$ is minimal. Any other disc has a corresponding 1-handle with both ends attached to non-spherical boundary 
components of chunks.

We can cut along all the discs which correspond to 1-handles with both ends in $S$, and so we see that $N$ can be thought of as a simpler manifold with boundary including $S$, with one or more 1-handles attached to $S$.

Step 3: Cutting along the separating 2-spheres.

This needs little attention for the moment. Note that cutting along such a 2-sphere corresponds to decomposing as a connected sum (up to adding or removing 3-balls, anyway).

Step 4: Cutting along the remaining discs.

As we noted in step 2, all such discs must correspond to 1-handles with both ends in non-spherical boundary components of chunks. Of course in particular this means that these 1-handles are not attached to simply connected chunks.

We are now ready to reverse the decomposition process. Let $K$ denote the disjoint union of the non simply connected chunks $C_{i}$. Then any core, up to almost homeomorphism, is obtained from $K$ by adding 1-handles, $S^{2} \times I^{\prime}$ 's and also simply connected compact manifolds. Eventually we construct a compact 3-manifold from $K$ by reversing steps one to four of the decomposition process.

We consider the steps of the decomposition individually, and in reverse order. To start with, $K$ is uniquely determined up to almost homeomorphism. At each stage, we must ensure that there are only finitely many possibilities for $K$, up to almost homeomorphism.

Step 4:

To reverse this, we add 1-handles to $K$. Let $H$ be such a 1-handle. Each end of $H$ is connected to a non spherical boundary component of a non simply connected chunk. Thus we do not need to consider simply connected chunks at this stage.

There are only $s$ non-simply connected chunks in $K$, and each chunk has only finitely many boundary components, and so there are only finitely many ways to attach each end of $H$, and hence there are only finitely many ways of attaching $H$ to $K$, since the ends of $H$ and the orientation of $H$ are the only factors in determining the homeomorphism class of the result. Note that we have used the fact that $H$ must be connected to non-spherical boundary components of chunks, and thus it is irrelevant that $K$ is defined only up to almost homeomorphism.

Step 3:

In this step, we add $S^{2} \times I^{\prime}$ 's to $K$, but since one end of an $S^{2} \times I$ can be attached to a simply connected chunk, we must also add these. Remember 
that all the $S^{2} \times I$ 's in this stage connect different components of $K$, and the end result of this is connected.

Consider an $S^{2} \times I$ with either (or both) ends attached to a simply connected chunk. Then it corresponds to forming a connected sum, with one of the summands being a simply connected compact 3-manifold. Thus, up to almost homeomorphism, we have done nothing.

Now we consider $S^{2} \times I^{\prime}$ 's which connect non simply connected chunks. Adding such an $S^{2} \times I$ corresponds to forming a connected sum, since each end of the $S^{2} \times I$ is connected to a different component of $K$. If $K$ has $n$ non simply connected components, then we must add $n-1 S^{2} \times I$ 's in this step, and however we do this, the result is unique up to almost homeomorphism. In particular, it is irrelevant which 2-sphere boundary components of $K$ we choose to attach an $S^{2} \times I$ to, and also it is irrelevant which components of $K$ the $S^{2} \times I$ is attached to, since the end result of step 3 is a connected manifold.

Step 2:

In this step, we attach 1-handles which have both ends in the same spherical boundary component of a chunk. Note that if we wish to add one such 1-handle, we have a choice of 2-sphere boundary components on which to attach it, but, assuming orientability, any choice gives the same result up to homeomorphism. If we are adding many 1-handles, we do not care which 2-spheres we attach them to, but only which 1-handles we allow to share the same boundary 2-spheres, which is a combinatorial question.

If we allow non-oriented 1-handles, there are more possibilities, but there are still only finitely many different ways of adding the 1-handles to $K$, up to almost homeomorphism of the resulting manifold.

Step 1:

As we noted earlier, this is equivalent to forming a connected sum with 2sphere bundles over the circle, up to almost homeomorphism. Each 2-sphere bundle could be orientable or non-orientable.

We have $\pi_{1}(M)=G=F_{r} * G_{1} * G_{2} * \cdots * G_{s}$. Thus there are $(r+s-1)$ 1-handles, non-trivial $S^{2} \times I$ 's and 2-sphere bundles over the circle to be added to the chunks $C_{\imath}$ to get a compact connected manifold with fundamental group $G$. Hence each of the steps one to four must terminate in fewer than $r+s-1$ steps, since the 1-handles, $S^{2} \times I$ 's and $S^{2}$-bundles added in these steps are precisely those needed to get a compact manifold with fundamental group $G$.

So after $r+s-1$ stages, and with a finite number of possibilities at each stage, we have a compact manifold with fundamental group $G$. Thus there are, up to almost homeomorphism, only finitely many compact 3-manifolds 
that can be formed from the $C_{i}$ with fundamental group $G$, and so $M$ can have only finitely many different cores up to almost homeomorphism. This completes the proof of Theorem 2.1.

\section{$\S 3$. Applications of uniqueness of cores.}

In this section we use Theorem 2.1 together with results of McCullough [McC] on compact cores to deduce information about the boundary of a 3-manifold $M$ with finitely generated fundamental group.

The first result is an obvious deduction from Theorem 2.1.

Corollary 3.1. Let $M$ be a 3-manifold with finitely generated group, and let $X_{i}$ be an infinite sequence of cores of $M$. Then there is a subsequence $X_{j}$ of $X_{i}$ such that all members of the sequence $X_{j}$ are almost homeomorphic.

Before we get to the main result of this section, we need a definition. Let $M$ be a 3-manifold with finitely generated fundamental group $G$. Let $F$ be a component of $\partial M$, and let $H=\operatorname{Im}\left(\pi_{1}(F) \rightarrow \pi_{1}(M)\right)$ be the image of the fundamental group of $F$ under the natural induced map into $G$. Then $H$ is finitely generated, by the result of Jaco in [Ja]. Now we can take a compact regular neighborhood of based loops in $F$ representing the generators of $H$, and add compressing discs in $F$ to get a compact subsurface $C$ of $F$ with $\operatorname{Im}\left(\pi_{1}(c) \rightarrow \pi_{1}(M)\right)=H$, and $C$ incompressible in $F$. Then:

Definition. With $M, F$ and $C$ as above, we call $C$ an essential core for $F$. Remark. $C$ need not be incompressible in $M$. Also, if $\operatorname{Im}\left(\pi_{1}(F) \rightarrow \pi_{1}(M)\right)$ is infinite cyclic, we can choose a simple closed curve on $F$ to represent a generator, and thus we may choose the essential core in this case to be an annulus or a Möbius band. We assume in what follows that we always choose such an essential core when possible.

We can now state the main result of this section. McCullough gives a result equivalent to part (i) and (ii) of this theorem in the case when $\partial M$ is incompressible as a corollary to his main theorem in $[\mathbf{M c C}]$. See also $[\mathbf{R - S}]$.

Theorem 3.2. Let $M$ be a 3-manifold with finitely generated group. Then:

(i) There are only finitely many boundary components $F$ of $M$ with $\operatorname{Im}\left(\pi_{1}(F) \rightarrow \pi_{1}(M)\right)$ not trivial or infinite cyclic,

(ii) There are only finitely many boundary components $F$ of $M$ with $\operatorname{Im}\left(\pi_{1}(F) \rightarrow \pi_{1}(M)\right)$ infinite cyclic and with essential core a Möbius band,

(iii) Of those components of the boundary $F_{i}$ with $\operatorname{Im}\left(\pi_{1}\left(F_{i}\right) \rightarrow \pi_{1}(M)\right)$ infinite cyclic and with essential core an annulus, there are only finitely many conjugacy classes in $\pi_{1}(M)$ of $\operatorname{Im}\left(\pi_{1}\left(F_{i}\right) \rightarrow \pi_{1}(M)\right)$. 
Proof. Let the boundary components of $M$ be $F_{i}$. Then we can find essential cores $C_{i}$ for the $F_{i}$, with the $C_{i}$ being annuli or Möbius bands when possible. We are only interested in those $F_{i}$ with non-trivial image in $\pi_{1}(M)$, so we will assume that none of the $C_{\imath}$ are discs. Now the theorem of McCullough [McC] tells us that given a (possibly disconnected) compact subsurface $C$ of the boundary of a manifold $M$, we can find a compact core $X$ for $M$ with $X \cap \partial M=C$. Thus a manifold $M$, we can find a sequence of cores $X_{i}$ for $M$ with $X_{i} \cap \partial M=\bigcup_{j=1}^{i} C_{j}$.

By taking a subsequence if necessary, we can assume that this sequence is stable, i.e. that all the $X_{i}$ are almost homeomorphic. Let $d X_{i}$ denote the union of all the non-spherical boundary components of $X_{i}$. Then $d X_{i}$ is homeomorphic to a fixed closed surface $d X$, for all $i$. Now for any union of non-trivial essential cores of boundary components of $M$, we can find an embedding of these essential cores in $d X_{i}$, for some $i$, and hence an embedding in $d X$. Since $d X$ has only finitely many components, we immediately see that there can only be a finite number of closed surfaces embedded in $d X$. So we are only concerned with subsurfaces of $d X$ which are not closed. Also we can assume that the subsurfaces are injective in $d X$, by adding discs lying in $d X$ to them. Thus none of the subsurfaces is a disc, and none of the components of the complement of the subsurfaces is a disc.

Assume for the moment that $d X$ is connected. Consider its Euler characteristic $\chi(d X)$. Any collection of embedded disjoint subsurfaces $\left\{C_{i}: i \leq m\right\}$ of $d X$ splits $d X$ into a collection of subsurfaces $\left\{C_{i}: i \leq n\right\}$ where $\left\{C_{i}: m+1 \leq i \leq n\right\}$ are the components of the complement of $\left\{C_{i}: i \leq m\right\}$. Then $\chi(d X)=\sum_{i=1}^{n} \chi\left(C_{i}\right)$. None of the $C_{i}$ are discs (or are closed), so $\chi\left(C_{\imath}\right) \leq 0 \forall i$. Also, any $C_{i}$ which are not Möbius bands or annuli have negative Euler characteristic, and so $d X$ can contain at most $|\chi(d X)|$ such surfaces. Of course, when $d X$ is not connected this holds for any component of $d X$. This proves the first part of the theorem.

Similarly $d X$ can contain only a finite number of Möbius bands, this time limited by the rank of $H_{1}\left(d X, \mathbb{Z}_{2}\right)$, and so also there are only a finite number of boundary components of $M$ with essential core a Möbius band. This completes part two of the theorem.

Consider now essential cores which are annuli. There is no bound on the number of these that can be embedded in a surface $d X$. However, we can embed only finitely many non-parallel such annuli. Parallel annuli have fundamental groups which are conjugate in $\pi_{1}(M)$, so this completes the third part of the theorem. 


\section{References}

[BJS] M.Brin, K. Johannson and G.P. Scott, Totally peripheral 3-manifolds, Pacific J. Math., 118 (1985), 37-51.

[H-S] L. Harris and P. Scott, Non-compact totally peripheral 3-manifolds, to appear in Pacific J. Math.

[Ja] W. Jaco, Lectures on 3-manifold topology, C.B.M.S. Regional conference series in mathematics number 43 .

[McC] D. McCullough, Compact submanifolds of 3-manifolds with boundary, Quart. J. Math Oxford (2), 37 (1986), 299-307.

-Mi-Sw] D. McCullough, A. Miller and G.A. Swarup, Uniqueness of cores of non-compact 3-manifolds, J. London. Math. Soc. (2), 32 (1985), 548-556.

[R-S] J.H. Rubinstein and G.A. Swarup, On Scott's core theorem, Bull London Math. Soc., 22 (1990), 495-498.

[Sc1] G.P. Scott, compact submanıfolds of 3-manifolds, J. London Math. Soc. (2), 7 (1973), 246-250.

[Sc2] fundamental groups of non-compact 3-manifolds, Proc. London Math. Soc. (3), 34 (1977), 303-326.

Received February 15, 1993 and revised July 25, 1993. The second author partially supported by NSF grant DMS 90-03974.

UNIVERSITY OF MiCHIGAN

AnN ARBor, MI 48109

E-mail address: pscott@umich.edu 



\section{PACIFIC JOURNAL OF MATHEMATICS}

Volume $172 \quad$ No. $1 \quad$ January 1996

A class of incomplete non-positively curved manifolds

BRIAN BOWDITCH

The quasi-linearity problem for $C^{*}$-algebras

41

L. J. BUNCE and JOHN DAVID MAITLAND WRIGHT

Distortion of boundary sets under inner functions. II

Jose Luis Fernandez Perez, Domingo Pestana and José Rodríguez

Irreducible non-dense $A_{1}^{(1)}$-modules

VJACHESLAV M. FUTORNY

$M$-hyperbolic real subsets of complex spaces

101

Giuliana Gigante, Giuseppe Tomassini and Sergio Venturini

Values of Bernoulli polynomials

ANDREW GRANVILLE and ZHI-WEI SUN

The uniqueness of compact cores for 3-manifolds

LUKE HARRIS and PETER SCOTT

Estimation of the number of periodic orbits

BOJU JIANG

Factorization of $p$-completely bounded multilinear maps

Christian Le MERdy

Finitely generated cohomology Hopf algebras and torsion

JAMEs Peicheng LiN

The positive-dimensional fibres of the Prym map

JUAN-CARLOS NARANJO

Entropy of a skew product with a $Z^{2}$-action

KYEWON KOH PARK

Commuting co-commuting squares and finite-dimensional Kac algebras

TAKASHI SANO

Second order ordinary differential equations with fully nonlinear two-point boundary 255 conditions. I

H. BEVAN THOMPSON

Second order ordinary differential equations with fully nonlinear two-point boundary conditions. II

H. BEVAN THOMPSON

The flat part of non-flat orbifolds

FENG XU 